Journal of Computer Science 3 (3): 174-179, 2007

ISSN 1549-3636

(C) 2007 Science Publications

\title{
Neural Networks for Interferences Suppression in DS/CDMA with Rayleigh Fading Channel and Power Control Error
}

\author{
${ }^{1}$ Y. Jabrane, ${ }^{1}$ R. Iqdour, ${ }^{1}$ B. Ait Essaid, ${ }^{2}$ N. Naja and ${ }^{3}$ S. Soukri \\ ${ }^{1}$ Department of Physics, Cadi Ayyad University, Faculty of Sciences Semlalia, \\ Avenue Prince My Abdellah, P.O. Box 2390, 40001, Marrakesh, Morocco \\ ${ }^{2}$ National Institute of Poste and Telecommunications, Av Allal Al Fassi- Madinat Al Irfane \\ Rabat-Instituts, Morocco \\ ${ }^{3}$ Motorola, Agdal, Rabat, Morocco
}

\begin{abstract}
The exponential chip weighting waveforms have been designed with the purpose of multiple access interferences (MAI) rejection based on the concept that the optimum despreading sequence in reference emphasizes the transitions in the received spreading signal of interest. The despreading sequence weighted by exponential chip waveforms was determined by only one parameter. The objective of this study, was to introduce neural networks (NN) to facilitate the computing of the bit error rate (BER) performance of a direct sequence code division multiple access (DS/CDMA) system over a Rayleigh multipath fading with power control error, for both coherent and noncoherent receivers, by producing the despreading sequences weighted (WDS) by exponential chip weighting waveforms. Numerical results show that the parameter values of the exponential chip weighting waveforms produced by the proposed network are nearly optimal and satisfactory in viewpoint of the achieved bit error rate (BER) performance.
\end{abstract}

Key words: Multiple access interferences, code division multiple access, weighted despreading sequences, neural networks, rake receiver, bit error rate

\section{INTRODUCTION}

The major limitation in the bit error rate (BER) and system performance and hence capacity, is due to multipath fading, (MAI) and imperfect power control. With the objective of MAI rejection, most of works had been made on CDMA performance in presence of multipath fading and are based on the fact that power control is perfect ${ }^{[1-3]}$, which is not really possible in practice.

In a previous work $^{[4]}$, the BER performance of a DS/CDMA system was analyzed with power control error over a multipath Rayleigh fading channel using both coherent and noncoherent receptions, the receivers under consideration employ the despreading sequences weighted (WDS) by adjustable exponential chip waveforms optimized for MAI rejection. The chip weighting waveforms employed are determined by only one parameter that leads to easy tuning of the waveforms in practice to achieve the best performance.
It is useful to note that the derived closed-form solutions, which enable to calculate de BER in ${ }^{[4]}$, require perfect definition of the spreading sequences for the signals of all users. Also, a number of computational efforts are needed to define the parameters of each spreading sequence.

In this work, Our objective is to reduce the computational intensity and shorten the process time to obtain optimal values of the parameter by using the learning ability and the high-speed computational capacity features of neural networks. Throughout the study, we have assumed that a receiver in the DS/CDMA system is perfectly capable of regenerating the reference spreading codes corresponding to each of the users' transmissions.

\section{System description}

Transmitter model: From the transmitter model presented in $^{[4]}$ which suppose that there are $\mathrm{K}$ users sharing the channel in a DS/CDMA system, the transmitted signal by the $k$ th user is given by :

Corresponding Author: $\quad$ Y. Jabrane, Department of Physics, Cadi Ayyad University, Faculty of Sciences Semlalia, Avenue Prince My Abdellah, P.O. Box 2390, 40001, Marrakesh, Morocco, Tel: 0021224434649, Fax: 0021224437410 
$\mathrm{S}_{\mathrm{k}}(\mathrm{t})=\sqrt{2 \mathrm{P}} \mathrm{G}_{\mathrm{k}} \mathrm{b}_{\mathrm{k}}(\mathrm{t}) \mathrm{a}_{\mathrm{k}}(\mathrm{t}) \cos \left(\omega_{0} \mathrm{t}+\theta_{\mathrm{k}}\right)$

Where $\mathrm{P}$ and $\omega_{0}$, common to all users, are the transmitted power and the carrier frequency, respectively and $\theta_{\mathrm{k}}$ is the phase introduced by the kth modulator. The parameter $G_{k}$ represents the power control error for the kth user and is modeled as a random variable uniformly distibuted in $\left[1-\varepsilon_{\mathrm{m}}, 1+\varepsilon_{\mathrm{m}}\right]$ where $\varepsilon_{\mathrm{m}}$ represents the maximum value of power control error for all users. $a_{k}(t)$ and $b_{k}(t)$ are the spreading sequences and the binary data sequences for the $k$ th user, respectively, they are given by:

$$
\begin{aligned}
& \mathrm{a}_{\mathrm{k}}(\mathrm{t})=\sum_{\mathrm{j}=\infty}^{\infty} \mathrm{a}_{\mathrm{j}}^{(\mathrm{k})} \mathrm{P}_{\mathrm{T}_{\mathrm{c}}}\left(\mathrm{t}-\mathrm{T}_{\mathrm{c}}\right) \\
& \mathrm{b}_{\mathrm{k}}(\mathrm{t})=\sum_{\mathrm{j}=\infty}^{\infty} \mathrm{b}_{\mathrm{j}}^{(\mathrm{k})} \mathrm{P}_{\mathrm{T}_{\mathrm{c}}}\left(\mathrm{t}-\mathrm{T}_{\mathrm{b}}\right)
\end{aligned}
$$

Where $\mathrm{T}_{\mathrm{c}}$ and $\mathrm{T}_{\mathrm{b}}$ as the chip and data durations, respectively and $\mathrm{P}_{\mathrm{x}}(\mathrm{y})=1$ for $0 \prec \mathrm{x} \prec \mathrm{y}$ and zero otherwise. $a_{j}^{(k)}$ and $b_{j}^{(k)}$ take the values +1 and -1 randomly and independantly with equal probabilities. It is assumed that the spreading sequence is periodic with period $N=\frac{T_{b}}{T_{c}}$ and $a_{j}^{(k)}=a_{j+N}^{(k)}$ for all $-\infty \prec j \prec+\infty$.

Channel model: In the following, it is assumed that the channel is frequency selective multipath for the uplink. The equivalent complex low-pass representation of the channel for the $k$ th user is given by:

$\mathrm{h}_{\mathrm{k}}(\mathrm{t})=\sum_{\mathrm{l}=0}^{\mathrm{L}_{\mathrm{p}}-l} \beta_{\mathrm{k} 1} \delta\left(\mathrm{t}-\tau_{\mathrm{kl}}\right) \mathrm{e}^{\mathrm{j} \eta_{\mathrm{k} 1}}$

Where random variables $\beta_{\mathrm{kl}}, \tau_{\mathrm{kl}}$ and $\eta_{\mathrm{k} 1}$ are the $l$ th path gain, delay and phase respectively, for the $k t h$ user. In $^{[4]}$, the following assumptions are considered:

For different users and pathsin each link, the random variables $\beta_{\mathrm{kl}}, \tau_{\mathrm{kl}}$ and $\eta_{\mathrm{kl}}$ are all statistically independent.

The random phases $\eta_{\mathrm{kl}}$ are uniformly distributed over $[0,2 \pi]$ and the path delays $\tau_{\mathrm{kl}}$ are uniformly distributed over $\left[0, \mathrm{~T}_{\mathrm{b}}\right]$.

There are $L_{p}$ paths for each user and these different paths are separated in time from each other by more than $2 \mathrm{~T}_{\mathrm{c}}$.
For each user, the path gain $\beta_{\mathrm{kl}}$ is a random variable with Rayleigh distibution given by:

$$
\mathrm{p}\left(\beta_{\mathrm{k} 1}\right)= \begin{cases}\frac{\beta_{\mathrm{kl}}}{\rho_{\mathrm{k} 1}} \exp \left(\frac{-\beta_{\mathrm{kl}}{ }^{2}}{2 \rho_{\mathrm{k} 1}}\right), & \beta_{\mathrm{kl}} \geq 0 \\ 0, & \beta_{\mathrm{kl} \prec 0}\end{cases}
$$

The fading rate in the channel is slow compared to the bit rate, so that the random parameters associated. with the channel do not vary significantly over tow consecutive bit intervals.

The received signal at the central station $r(t)$, mixed with AWGN $n(t)$ with two sided spectral density $\frac{\mathrm{N}_{0}}{2}$, is given by:

$$
\begin{aligned}
& \mathrm{r}(\mathrm{t})=\sqrt{2 \mathrm{P}} \sum_{\mathrm{k}=1}^{\mathrm{K}} \sum_{\mathrm{l}=0}^{\mathrm{L}_{\mathrm{p}}-1} \mathrm{G}_{\mathrm{k}} \beta_{\mathrm{kl}} \mathrm{a}_{\mathrm{k}}\left(\mathrm{t}-\tau_{\mathrm{kl}}\right) \mathrm{b}_{\mathrm{k}}\left(\mathrm{t}-\tau_{\mathrm{kl}}\right) \\
& \cos \left(\omega_{0} \mathrm{t}+\Phi_{\mathrm{kl}}\right)+\mathrm{n}_{\mathrm{c}}(\mathrm{t}) \cos \left(\omega_{0} \mathrm{t}\right)+\mathrm{n}_{\mathrm{s}}(\mathrm{t}) \sin \left(\omega_{0} \mathrm{t}\right)
\end{aligned}
$$

Where $\Phi_{\mathrm{kl}}=\theta_{\mathrm{kl}}+\eta_{\mathrm{kl}}-\omega_{0} \tau_{\mathrm{kl}}$ and the term $\mathrm{n}_{\mathrm{c}}(\mathrm{t})$ et $\mathrm{n}_{\mathrm{s}}(\mathrm{t})$ are low-pass equivalent components of the AWGN n(t).

Receiver model: For BPSK modulation, ${ }^{[4]}$ described the structure of one of the paths of a RAKE receiver using coherent detection:

In the goal to reject MAI, a bank of single path matched filters, each of which is matched to different paths, have the same impulse response matched to $2 \hat{\mathrm{a}}_{\mathrm{k}}(\mathrm{t}) \cos \left(\omega_{0} \mathrm{t}\right) \mathrm{P}_{\mathrm{T}_{\mathrm{b}}}(\mathrm{t})$ where $\hat{\mathrm{a}}_{\mathrm{k}}(\mathrm{t})$ is the weighted despreading sequence with details given below. The outputs of all single matched filters $\xi_{\mathrm{kl}}(\kappa)$, $1 \in\left[0, L_{R}-1\right]$, where $L_{R}$ is the order of diversity, are weighted the corresponding path gains and then summed to form a single decision variable $\xi_{\mathrm{k}}(\kappa)$. The weighted despreading function of the $k t h$ user's RAKE receiver can be expressed as:

$$
\begin{aligned}
& \hat{a}_{k}(t)=\sum_{j=-\infty}^{\infty} \begin{array}{cc}
(k) & (k) \\
a & j
\end{array}\left(t-j T_{c} /\left\{\begin{array}{cc}
(k) & (k) \\
c & c \\
j & j+1
\end{array}\right\}\right) \\
& \underset{\mathrm{j}}{(\mathrm{k})} \underset{\mathrm{j}-1}{\mathrm{c}} \underset{\mathrm{j}}{(\mathrm{k})(\mathrm{k})} \stackrel{(\mathrm{k})}{\mathrm{a}} \underset{\mathrm{j}}{\mathrm{w}}\left(\mathrm{t} /\left\{\begin{array}{c}
(\mathrm{k})(\mathrm{k}) \\
\mathrm{c}, \underset{\mathrm{j}+1}{\mathrm{c}}
\end{array}\right\}\right) \text { for } 0 \leq \mathrm{t} \leq \mathrm{T}_{\mathrm{c}}, \text { is }
\end{aligned}
$$

the $j$ th chip weighting waveforms for the $k$ th receiver conditioned on the status of three consecutive chips $\left\{\begin{array}{c}(\mathrm{k})(\mathrm{k}) \\ \mathrm{c}, \underset{\mathrm{j}}{\mathrm{j}+1}\end{array}\right\}=\left\{\begin{array}{cccc}(\mathrm{k})(\mathrm{k}) & (\mathrm{k})(\mathrm{k}) \\ \mathrm{a} & \mathrm{a} & \mathrm{a} & \mathrm{a} \\ \mathrm{j}-1 & \mathrm{j} & \mathrm{j} & \mathrm{j}+1\end{array}\right\} \quad$ and $\quad \mathrm{P}_{\mathrm{x}}(\mathrm{y})=1 \quad$ for 
$0 \prec \mathrm{y} \prec \mathrm{x}$ and 0 otherwise. The $j$ th chip conditional weighting waveforms for the $k$ th receiver is defined by ${ }^{[1]}$ as:

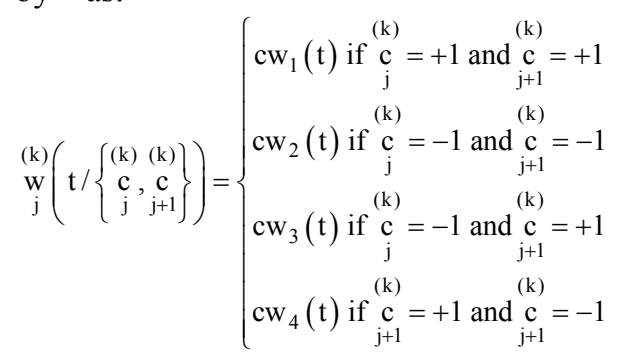

The elements of the chip weighting waveform vector $\left\{\mathrm{cw}_{1}(\mathrm{t}), \mathrm{cw}_{2}(\mathrm{t}), \mathrm{cw}_{3}(\mathrm{t}), \mathrm{cw}_{4}(\mathrm{t})\right\}$ are given by:

$$
\left\{\begin{array}{l}
\mathrm{cw}_{1}(\mathrm{t})=\exp \left(-\frac{\gamma}{2}\right) \mathrm{P}_{\mathrm{T}_{\mathrm{c}}}(\mathrm{t}) \\
\mathrm{cW}_{2}(\mathrm{t})=\exp \left(-\gamma \frac{\mathrm{t}}{\mathrm{T}_{\mathrm{c}}}\right) \mathrm{P}_{\frac{\mathrm{T}_{\mathrm{c}}}{2}}(\mathrm{t})+\exp \left(-\gamma\left[1-\frac{\mathrm{t}}{\mathrm{T}_{\mathrm{c}}}\right]\right) \mathrm{P}_{\frac{\mathrm{T}_{\mathrm{c}}}{2}}\left(\mathrm{t}-\frac{\mathrm{T}_{\mathrm{c}}}{2}\right) \\
\mathrm{cW}_{3}(\mathrm{t})=\exp \left(-\gamma \frac{\mathrm{t}}{\mathrm{T}_{\mathrm{c}}}\right) \mathrm{P}_{\mathrm{T}_{\mathrm{c}}}(\mathrm{t})+\exp \left(-\frac{\gamma}{2}\right) \mathrm{P}_{\frac{\mathrm{T}_{\mathrm{c}}}{2}}\left(\mathrm{t}-\frac{\mathrm{T}_{\mathrm{c}}}{2}\right) \\
\mathrm{cw}_{4}(\mathrm{t})=\exp \left(-\frac{\gamma}{2}\right) \mathrm{P}_{\frac{\mathrm{T}_{\mathrm{c}}}{2}}(\mathrm{t})+\exp \left(-\gamma\left[1-\frac{\mathrm{t}}{\mathrm{T}_{\mathrm{c}}}\right]\right) \mathrm{P}_{\frac{\mathrm{T}_{\mathrm{c}}}{2}}\left(\mathrm{t}-\frac{\mathrm{T}_{\mathrm{c}}}{2}\right)
\end{array}\right.
$$

$\gamma \in[0, \infty]$ is the parameter of the chip weighting waveforms. For DPSK modulation, the structure of path 1 of $k$ th user's RAKE demodulator using noncoherent detection is described $\mathrm{in}^{[4]}$ :

In this case, the outputs of all single path receiver $\mathfrak{R e}\left[\vartheta_{\mathrm{kl}}(\kappa) \vartheta_{\mathrm{kl}}^{*}(\kappa-1)\right]$ summed to form a single decision variable $\vartheta_{\mathrm{k}}(\kappa)$.

System performance: The evaluation of the system performance requires calculating the bit error rate (BER). $\mathrm{In}^{[4]}$ a detailed analyses give the BER expressions for coherent ${ }^{[4]}$, eq. (38)) and noncoherent $\left({ }^{[4]}\right.$, eq. (55)) reception:

For coherent reception:

$$
\mathrm{Pe}^{(\mathrm{k})}=\frac{1}{2}-\sum_{\mathrm{d}=1}^{\mathrm{L}_{\mathrm{R}}} \frac{\left(\begin{array}{c}
2 \mathrm{~d}-2 \\
\mathrm{~d}-1
\end{array}\right)}{2^{2 \mathrm{~d}} \varepsilon_{\mathrm{m}} \hat{\mathrm{H}}(2 \mathrm{~d}-3)} \times\left\{\left(\mathrm{b}^{2}+1\right)^{\frac{3-2 \mathrm{~d}}{2}}-\left(\mathrm{a}^{2}+1\right)^{\frac{3-2 \mathrm{~d}}{2}}\right\}
$$

For noncoherent reception:

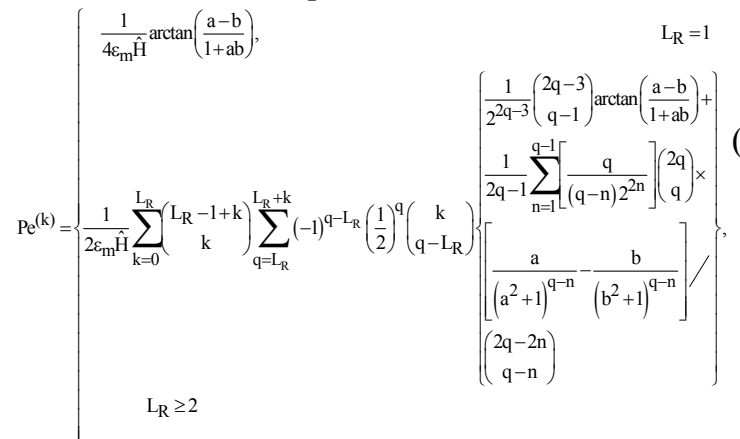

Where $a=\left(1+\varepsilon_{\mathrm{m}}\right) \hat{\mathrm{H}}, \quad \mathrm{b}=\left(1-\varepsilon_{\mathrm{m}}\right) \hat{\mathrm{H}}$ and $\hat{\mathrm{H}}$ is the average signal to interference plus noise ratio per channel, given by $\left({ }^{[4]}\right.$, eq. (36)):

$$
\hat{\mathrm{H}}=\left\{\begin{array}{c}
\frac{\gamma\left[\chi\left(1-\mathrm{e}^{-\gamma}\right)+\gamma(1-\chi) \mathrm{e}^{-\gamma}\right]}{\mathrm{k}_{\mathrm{b}}\left[2 \chi\left(1-\mathrm{e}^{-\gamma / 2}\right)+\gamma(1-\chi) \mathrm{e}^{-\gamma / 2}\right]^{2}} \\
+\frac{\left(\mathrm{KL}_{\mathrm{p}}-1\right)\left(1+\frac{\varepsilon_{\mathrm{m}}{ }^{2}}{3}\right) \mathrm{E}\left(\Gamma\left\{\begin{array}{c}
(\mathrm{k}) \\
\mathrm{c} \\
\mathrm{j}
\end{array}\right\}, \gamma\right)}{\mathrm{N}\left[2 \chi\left(\mathrm{e}^{\gamma / 2}-1\right)+\gamma(1-\chi)\right]^{2}}
\end{array}\right\}^{-\frac{1}{2}}
$$

Where, $\gamma$ is the parameter of the exponential chip weighting waveforms tuned to maximize $\hat{\mathrm{H}}, \mathrm{K}$ is the number of active users, $\mathrm{k}_{\mathrm{b}}$ is the signal to noise ratio, $\chi=\hat{\mathrm{N}}_{\mathrm{k}} / \mathrm{N}, \quad \mathrm{N}_{\mathrm{k}}$ is a random variable which represents the number of occurrences of $\underset{j}{\stackrel{(k)}{c}}=-1$ for all $\mathrm{j} \in[0, \mathrm{~N}-1]$ and the term $\mathrm{E}\left(\Gamma\left\{\mathrm{c}_{\mathrm{j}}^{(\mathrm{k})}\right\}, \gamma\right)$ is given by:

$$
\begin{aligned}
& \int \stackrel{(\mathrm{k})}{\Gamma}[-1,-1,-1\}\left[4+\frac{12}{\gamma}-\frac{16 \mathrm{e}^{\gamma / 2}}{\gamma}+\frac{4 \mathrm{e}^{\gamma}}{\gamma}\right] \\
& +\left(\begin{array}{c}
\stackrel{(\mathrm{k})}{\Gamma}+-1,-1,1\} \\
\left.\left\{\begin{array}{l}
(\mathrm{k}) \\
\Gamma
\end{array}\right)-1,-1\right\}
\end{array}\right)\left[\frac{5}{2}-\frac{\gamma}{4}+\frac{\gamma^{2}}{24}+\frac{19}{2 \gamma}+\mathrm{e}^{\gamma / 2}-\frac{12 \mathrm{e}^{\gamma / 2}}{\gamma}+\frac{5 \mathrm{e}^{\gamma}}{2 \gamma}\right]+
\end{aligned}
$$

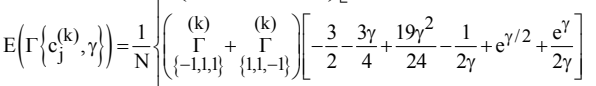

$$
\begin{aligned}
& +\stackrel{(\mathrm{k})}{\Gamma}[-1,1,-1\}\left[-3-\frac{3 \gamma}{2}+\frac{7 \gamma^{2}}{12}-\frac{1}{\gamma}+2 \mathrm{e}^{\gamma / 2}+\frac{\mathrm{e}^{\gamma}}{\gamma}\right]+ \\
& \underset{\{1,-1,1,\}]}{(\mathrm{k})}\left[1-\frac{\gamma}{2}+\frac{\gamma^{2}}{12}+\frac{7}{\gamma}+2 \mathrm{e}^{\gamma / 2}-\frac{8 \mathrm{e}^{\gamma / 2}}{\gamma}+\frac{\mathrm{e}^{\gamma}}{\gamma}\right]+\gamma^{2} \stackrel{(\mathrm{k})}{\underset{\{1,1,1\}}{(1)}}
\end{aligned}
$$

Where $\underset{\left\{\mathrm{v}_{1}, \mathrm{v}_{2}, \mathrm{v}_{3}\right\}}{\stackrel{(\mathrm{k})}{\Gamma}}$ is the number of occurrences of $\left\{\begin{array}{ccc}(k) & (k) & (k) \\ c & c & c \\ j-1 & j & j+1\end{array}\right\}=\left\{v_{1}, v_{2}, v_{3}\right\}$ for all $j \in[0, N-1]$ in the kth user's spreading sequence. Each element of $\left\{\mathrm{v}_{1}, \mathrm{v}_{2}, \mathrm{v}_{3}\right\}$ takes values +1 or -1 with equal probabilities.

A neural networks based determination: The most common neural network model is the Multi-Layered Perceptrons $\mathrm{MLP}^{[5]}$. This type of neural network is known as a supervised network because it requires a desired output in order to learn. The notation R-S1-S2$\mathrm{S}$ refers to a MLP with two hidden layers. The first layer have $\mathrm{R}$ neurons is called the input layer, the last is the output layer equipped with $\mathrm{S}$ neurons and the intermediate layers are the hidden layers with S1, S2 neurons. Each neuron of a layer is connected to all the neurons of the following layer (feed-forward neural network). 
We associate a weighting coefficient (synaptic weight) to each connection. These weights are stored in the matrices of weight noted W1, W2 and W3 (example : R-S1-S2-S network). The element $(i, j)$ of a weight matrix represents the connection weight connecting neuron $\mathrm{i}$ of the downstream layer to neuron $\mathrm{j}$ of the upstream layer.

Each layer (except that of input) is connected to a special cell with a constant output of value 1 . The corresponding weights are stored in a vector called bias and noted b1, b2 and b3 in the case of two hidden layers. Each neuron $\mathrm{i}$ of the first hidden layer computes his input net1[i] and his output (its activation) a1[i] as follow:

$\operatorname{net} 1[\mathrm{i}]=\prec \mathrm{W} 1[\mathrm{i}], \mathrm{p} \succ+\mathrm{bl}[\mathrm{i}]$

$\mathrm{a} 1[\mathrm{i}]=\mathrm{F} 1(\operatorname{net}[\mathrm{i}])$

Where $\mathrm{W} 1[\mathrm{i}]$ is the ith line of $\mathrm{W} 1,<>$ is the notation for the usual scalar product, $\mathrm{p}$ is the input vector and $\mathrm{F} 1$ is the activation function associates to the first hidden layer. The activation functions are non linear and of sigmoid type, i.e:

$\mathrm{F}(\mathrm{x})=\frac{1}{1+\mathrm{e}^{-\mathrm{x}}}$

The activations stored in the vector al are propagated to the cells of the following layer. In a similar way, we calculate the second activation vector:

$\mathrm{a} 2[\mathrm{i}]=\mathrm{F} 2(\prec \mathrm{W} 2[\mathrm{i}], \mathrm{a} 1 \succ+\mathrm{b} 2[\mathrm{i}])$

This mechanism continues to the last layer and makes it possible to obtain the output vector $t$ corresponding to the input $\mathrm{p}$. The network inputs consist of vectors of size $\mathrm{R}$ stored in a matrix $\mathrm{P}$ with $\mathrm{N}$ columns. Each column $\mathrm{p}$ of $\mathrm{P}$ is associated a desired vector $\mathrm{q}$ of the output $\mathrm{Q}$ with size $\mathrm{S}$ stored in a matrix $\mathrm{Q}$. The outputs computed by the network are stored in a matrix $\mathrm{T}$.

The required goal, is the learning of associations $(p, q)$ : the network must restore the desired output $q$ (or an output rather close to $Q$ ) when the form $p$ is presented as an input. The training of the MLP networks consists in computing the weights of connections between the neurons in order to minimise a square criterion $\mathrm{E}$ :

$$
\begin{aligned}
& E=\frac{1}{N} \sum_{p=1}^{N} E_{p} \\
& E_{p}=\frac{1}{2} \sum_{j=1}^{s}(Q[j][p]-T[j][p])^{2}
\end{aligned}
$$

For the real applications, we do not know the pace of the error function $\mathrm{E}$ in the space of the weights, what has as consequence when the non-linear training algorithm converges, we are never assured that the obtained minimum is global.

Backpropagation algorithm was created by generalizing the Widrow-Hoff learning rule to multiplelayer networks and non-linear differentiable transfer functions. The standard backpropagation is a gradient descent algorithm in which the network weights are moved along the negative of the gradient of the performance function E. An iteration of this algorithm can be written:

$$
\mathrm{w}(\mathrm{k}+1)=\mathrm{w}(\mathrm{k})-\varepsilon \mathrm{g}_{\mathrm{w}}(\mathrm{k})
$$

Where:

$\mathrm{g}_{\mathrm{w}}(\mathrm{k})=\left(\frac{\partial \mathrm{E}}{\partial \mathrm{w}_{1}(\mathrm{k})}, \ldots \ldots \ldots ., \frac{\partial \mathrm{E}}{\partial \mathrm{w}_{\mathrm{n}}(\mathrm{k})}\right)^{\mathrm{T}}$

The gradient error evaluated in $\mathrm{w}(\mathrm{k}) . \mathrm{n}$ is the number of the connections of the network, $\mathrm{k}$ an index of the iteration, $\mathrm{w}(\mathrm{k})=\left(\mathrm{w}_{1}(\mathrm{k}), \ldots \ldots \ldots, \mathrm{w}_{\mathrm{n}}(\mathrm{k})\right)^{\mathrm{T}}$ is The weight vector in the iteration $\mathrm{k}$ and $\varepsilon$ is a learning rate $(\varepsilon \succ 0)$.

Parameter $\varepsilon$ regulates the size of the gradient step. The performance of the algorithm is very sensitive to the proper setting of the learning rate. If the learning rate is set too high, the algorithm may oscillate and becomes unstable. If the learning rate is too small, the algorithm will take too long to converge. The greatest disadvantage of this algorithm is that it does not even ensure convergence towards a local minimum ${ }^{[6]}$. Several algorithms are proposed to land to this problem. In this work, we have used the Levemberg-Marquardt algorithm $^{[7]}$ that combines the best features of GaussNewton and gradient descent method was used to estimate the parameters of the neural networks model (weights).

The neural networks employed has eight inputs and one output, seven of the inputs are bound directly to the used code:

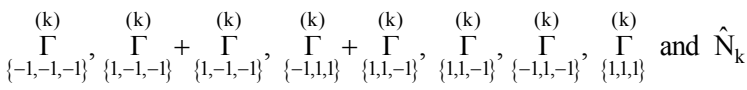

The last input is $s n r=k_{b}$ and the output of the neural networks model is $\gamma$. The $\mathrm{k}_{\mathrm{b}}$ values of training data have been taken from the range of $[0,22] \mathrm{dB}$.

\section{NUMERICAL RESULTS}

Here, we present the numerical results of our proposed method. The used codes in Table 1 are those of Gold having $N=31$ for their good correlation properties $^{[8,9]}$. The number of users is $K=9$, Table 2 
Table 1: $\quad$ Code of $\mathrm{N}=31$

Code $1 \quad 0110100101010110011101011111101$

Code 20111110011110111111001100001010

Code 30000100011101010010111100110110

Code $4 \quad 0000011101001000010011111111010$

Code $5 \quad 0011000001110010001010111101101$

Code $6 \quad 1100010110011011110100011001000$

Code $7 \quad 1001000100110100001000010101010$

Code $8 \quad 0010000111101010001101001001111$

Code $9 \quad 1001110111000001101000100101110$

Table 2: Quantities $\Gamma_{\left\{\mathrm{v}_{1}, \mathrm{v}_{2}, \mathrm{v}_{3}\right\}}^{(\mathrm{k})}$ and $\hat{\mathrm{N}}_{\mathrm{k}}$ of the code set having N=31

\begin{tabular}{|c|c|c|c|c|c|c|c|}
\hline Code & $\stackrel{\substack{\text { (k) } \\
\Gamma \\
\{-1,-1,-1\}}}{ }$ & $\begin{array}{c}\stackrel{(\mathrm{k})}{\Gamma} \\
\{-1,-1,1\} \\
\left\{\begin{array}{l}(\mathrm{k}) \\
\Gamma\end{array},-1,-1\right\}\end{array}$ & $\stackrel{(\mathrm{k})}{\Gamma}+\stackrel{(\mathrm{k})}{\Gamma_{\{-1,1,1\}}} \underset{\{1,1,-1\}}{ }$ & $\begin{array}{c}\stackrel{(\mathrm{k})}{\Gamma} \\
\{-1,1,-1\}\end{array}$ & 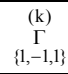 & $\begin{array}{l}(\mathrm{k}) \\
\Gamma \\
\{1,1,1\}\end{array}$ & $\hat{\mathrm{N}}_{\mathrm{k}}$ \\
\hline 1 & 10 & 8 & 4 & 4 & 2 & 3 & 20 \\
\hline 2 & 2 & 4 & 8 & 4 & 6 & 7 & 12 \\
\hline 3 & 4 & 8 & 8 & 4 & 4 & 3 & 16 \\
\hline 4 & 2 & 8 & 8 & 2 & 2 & 9 & 12 \\
\hline 5 & 4 & 8 & 8 & 4 & 4 & 3 & 16 \\
\hline 6 & 2 & 8 & 8 & 6 & 6 & 1 & 16 \\
\hline 7 & 9 & 10 & 6 & 3 & 1 & 2 & 20 \\
\hline 8 & 4 & 8 & 8 & 4 & 4 & 3 & 16 \\
\hline 9 & 3 & 10 & 10 & 3 & 3 & 2 & 16 \\
\hline
\end{tabular}

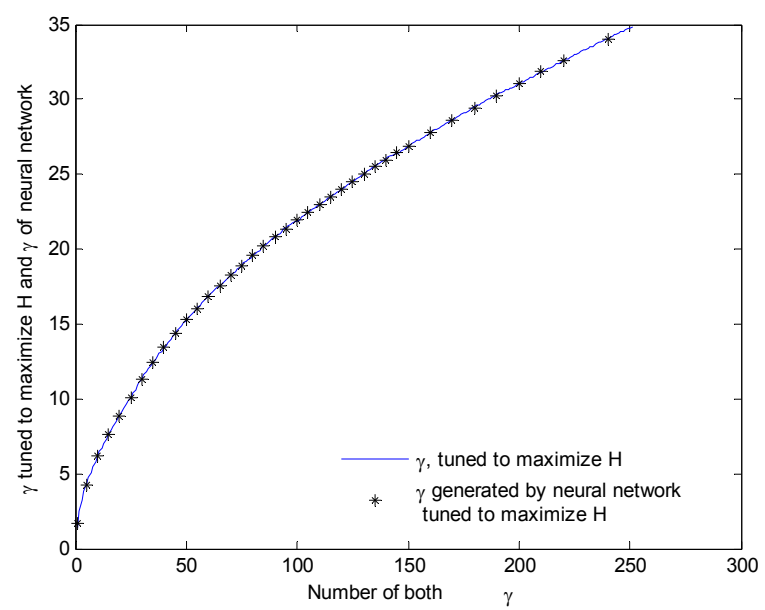

Fig. 1: The neural network model: $\gamma$ tuned to maximize $\hat{H}$ versus their number

gives $\Gamma_{\left\{\mathrm{v}_{1}, \mathrm{v}_{2}, \mathrm{v}_{3}\right\}}^{(\mathrm{k})}$ and $\hat{\mathrm{N}}_{\mathrm{k}}$ for each code. From these datas, code 1 is used as reference to train the neural networks model employed varying $\mathrm{snr}=\mathrm{k}_{\mathrm{b}}$. After learning, the neural networks model generalize the relation between $\gamma$ tuned to maximize $\hat{H}$ and the spreading codes varying snr (Fig. 1), it was tested with unseen snr values.

Figure 2 and 3 give the BER calculated by WDS generated by neural network model versus snr for various values $L_{R}=1,2,3,4$ respectively, for coherent and noncoherent reception when $\mathrm{L}_{\mathrm{P}=4} 4$ and $\varepsilon_{\mathrm{m}}=0$.

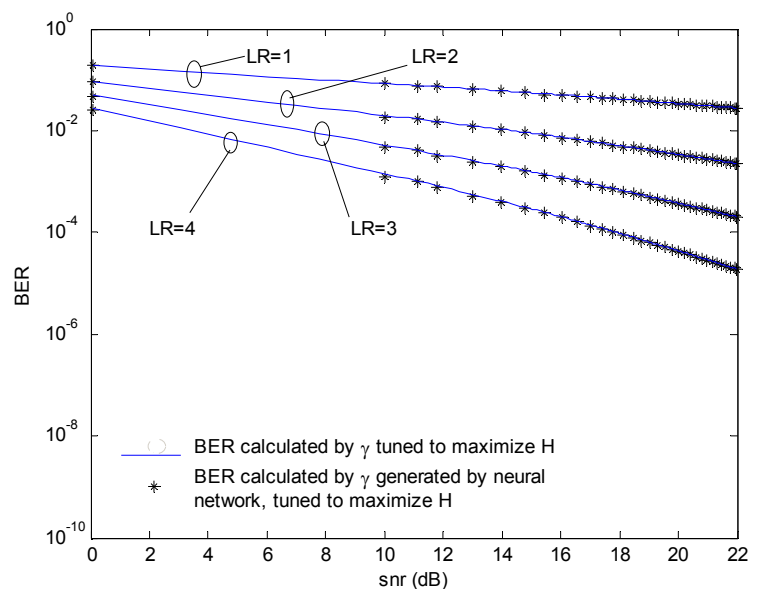

Fig. 2: BER calculated by WDS generated by neural network model versus snr for various values $\mathrm{L}_{\mathrm{R}}=1,2,3,4$ for coherent reception $\left(\mathrm{L}_{\mathrm{p}}=4, \varepsilon_{\mathrm{m}}=0\right)$

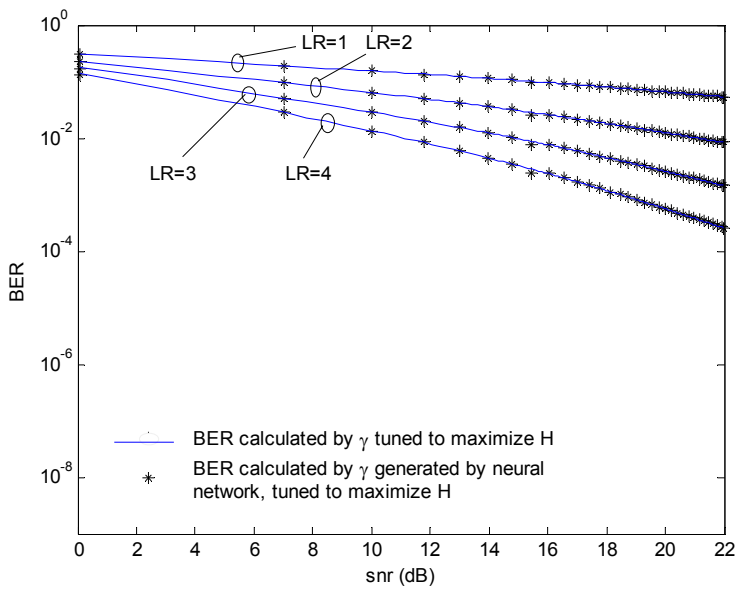

Fig. 3: BER calculated by WDS generated by neural network model versus snr for various values $\mathrm{L}_{\mathrm{R}}=1,2,3,4$ for noncoherent reception $\left(\mathrm{L}_{\mathrm{p}}=4, \varepsilon_{\mathrm{m}}=0\right)$

It is obvious that any performance degradation does not result from using the obtained values in place of the optimal values $(\gamma$ o maximize $\hat{\mathrm{H}})$, for these selected codes. Also, we tested the network with the other codes existing in the code set as a reference and see that it produces similar satisfactory results for those, too.

It is worth mentioning that all calculations took almost $0.1-0.4 \mathrm{sec}$ on a personal computer with a Pentium IV processor running at $2.8 \mathrm{GHz}$ to obtain a result against an arbitrary set of parameters. When the calculation time is considered for the optimal values, it can be said that the proposed approach is almost six 
times faster than the classical technique. As a result, the neural approach is efficient and useful for the determination process of the despreading sequences weighted by exponential chip waveforms.

\section{CONCLUSION}

A new approach based on $\mathrm{NN}$ has been successfully presented in this study to help the determination process of the exponentially weighted despreading sequences for a DS/CDMA system. In this approach, the nearly optimal values of the parameter are simply and quickly computed. Using the results computed by the neural approach does not cause any degradation on the performance of the system. Moreover, the approach presented in this study can be used for DS-CDMA systems having different processing gain and employ exponentially weighted despreading sequences in the receivers. Even if the training time takes a few seconds, the proposed method provides simplicity after training. Instead of complicated mathematical functions, there are a number of weight multiplication and summation in the structure of the neural model. So, the neural model is very simple and useful. The obtained neural structure can be easily implemented or inserted in a neural hardware available in the market. The neural hardware might be used not only in base stations but also for mobile units.

\section{REFERENCES}

1. Huang, Y. and T.S. Ng, 1999. A DS-CDMA system using despreading sequences weighted by adjustable chip waveforms. IEEE Trans. Commun., 47: 1884-1896.
2. Jabrane, Y., R. Iqdour, B. Ait Essaid and N. Naja, 2006. A simple and efficient procedure for calculating the tuning values of exponential chip weighting waveforms in DS/CDMA. Physical and Chemical News J., (In press).

3. Jabrane, Y., R. Iqdour, B. Ait Essaid and N. Naja, 2006. Determination of weighted despreading sequences for a DS/CDMA system using a new method. IEEE section, 4th Intl. Conf. JTEA 12-14 May, Tunisie.

4. Huang, Y. and T.S. Ng, 1999. DS-CDMA with power control error using weighted despreading sequences over a multipath Rayleigh fading channel. IEEE Trans.Veh.Technol., 48: 1067-1079.

5. Hopfield, J., 1982. Neural networks and physical systems with emergent collective computational abilities. Proc. of the Natl. Acd. Sci., 79: 25542558.

6. Iqdour, R. and A. Zeroual, 2006. The multi-layered perceptrons neural networks for the prediction of daily solar radiation. Intl. J. Signal Processing, World Enformatika Society, 3: 24-29.

7. Dayhoff, J., 1990. Neural Networks Architectures. Editions Van Norstrand Reynold.

8. Dinan, H,E. and B. Jabbari, 1998. Spreading codes for direct sequence CDMA and wideband CDMA cellular networks. IEEE Comm. Mag., 36: 48-54.

9. Kârkkainen, H.A. and K.P.A. Leppânen, 2000. The influence of initial-phases of a PN code set on the performance of an asynchronous DS/CDMA system. Wirless Pers. Commun., 13: 279-293. 